\title{
Evaluation of sexual dysfunction prevalence in infertile men with non-obstructive azoospermia
}

\author{
Taha Numan Yıkılmaz, Erdem Öztürk, Nurullah Hamidi, İsmail Selvi, Halil Başar, Levent Peşkircioğlu \\ Department of Urology, Dr. Abdurrahman Yurtaslan Oncology Training and Research Hospital, Ankara.
}

\begin{abstract}
Summary Objectives: To determine the prevalence of sexual dysfunction in male partners of infertile couples and evaluate the effect of childlessness on erectile dysfunction (ED) and sexual relationship stress. Materials and methods: We collected datas of couples who attended our clinics for infertility between 2009 and 2016. Erectile dysfunction was investigated with the Questionnaires of International Index of Erectile Function-15 (IIEF-15) whereas premature ejaculation (PE) status with the Premature Ejaculation Diagnostic Tool (PEDT). The stress status of the childlessness in terms of sexual intercourse was scored by the Visual analogue scale (VAS) questionnaire. These scores were measured before and after a successful assisted reproductive treatment with the birth of the child.

Results: The median age of the 193 male patients was 31 years (range 23-48). Erectile dysfunction was found in 68 (35.2\%) and PE in $42(21.7 \%)$ subjects. One hundred and forty-one couples were treated with assisted reproductive treatments. Forty eight couples had successful pregnancy. The IIEF-15 test was repeated after the birth of the child to the male partners of these couples. We observed that the IIEF-15 scores increased from 16 to $21(p=0.014)$. However there were no significant improvement on their ejaculation status $(p>0.05)$. The mean VAS scores of male partners was 5.2 (3-10) in the treatment period while it decreased to $4.1(0-8)$ after the birth of the chils $(p=0.02)$. Statistically analysis showed a correlation between VAS and infertility as did IIEF-15.

Conclusions: We observed that having children has a reducing effect on sexual relationship stress. Infertility is absolutely blamed on the women and men. This condition may have negative effects on male sexual performance and it is closely related with some emerging female sexual disorders. It should be taken into consideration that infertile couples may have sexual dysfunction.
\end{abstract}

KEY WORDS: Andrology; Infertility; Childlessness; Anxiety; Erectile dysfunction.

Submitted 12 May 2019; Accepted 2 August 2019

\section{INTRODUCTION}

Infertility is the inability to have child after 1 year of unprotected intercouse. Fifteen percent of couples experience difficulty conceiving a child. In between one-third and one-half of these, an abnormality can be found in the male partner (1). Infertility has been described as a stressor and a life crisis for individuals or couples, which results in a lower life quality and enhanced marital conflicts (2-5). These stresses play havoc with the couple's sex life. The physical health and emotional well-being of many individuals and couples of reproductive age are significantly affected by infertility. Sexual function is one of the important components of health and overall quality of life (6). Thus, couples with infertility may have abnormalities of sexual function, reduced sexual activity and this leads to an increase in the numbers of past years without a baby owner (7).

The relationship between sexual problems and infertility is unclear. Infertility causes many psychosexual problems such as loss of libido (with a consequent decrease in sexual activity), impotence, inhibition of orgasm and premature ejaculation (little or no control over ejaculatory response withnejaculation that may occur before vaginal entry achieved) or retarded ejaculation (difficulty ejaculating intravaginally, or at all) in male (8).

In contrast, sexual dysfunction may have an etiological role on infertility. Several studies from the United States (US) have suggested that infertility does not impact on sexual or erectile function after controlling for differences in intercourse frequency and/or socio-economic factors (9-11). One the other hand, many studies from US have suggested that infertility is often associated with sexual problems in men (12). The aim of this study is determining the prevalence of sexual dysfunction in male partners of infertile couples and evaluating the effect of childlessness on erectile dysfunction (ED) and sexual relationship stress in male partners.

\section{MATERIALS AND METHODS}

We collected data of couples who attended our clinics for infertility from 2009 to 2016. Age of couples, educational status of couples, duration of marriage, timing of obtaining first sexuality education, the number of successful or unsuccessful conception history of prior paternity, number of intercourse and sexual function were enrolled. Sexual function involved erectile dysfunction and premature ejaculation for men. Erectile dysfunction was investigated with the Questionnaires of International Index of Erectile Function-15 (IIEF-15) (11), in its Turkish translation.

Male participants were invited to complete several selfreported questionnaires including modified the IIEF-15 and the modified International Index of Erectile Function (IIEF-5) which consists 5 questions: 2 regarding erectile function, 1 concerning orgasmic function, 1 question on sexual desire, and 1 on satisfaction with intercourse. 
IIEF-15, a 15-item questionnaire for the evaluation of 5 domains of male sexual function (desire, erectile function, intercourse satisfaction, orgasmic function and overall satisfaction) $(13,14)$. The questionnaire investigated both spontaneous sex for pleasure and sex intended to lead to pregnancy. An IIEF-15 Erectile Function domain score less than 26 was used as a cut-off for the presence of erectile dysfunction (15). According to IIEF15, score was categorized as: (score 6-10) severe ED; (score 11-16) moderate ED; (score 17-21) mild to moderate ED; (score 22-25) mild ED; (score 26-30) no ED.

Premature ejaculation (PE) was determined by a questionnaire consisting of 5 separate questions called Premature ejaculation diagnostic tool (PEDT) (16). A PEDT score less than 8 indicates no PE, on the contrary PE was diagnosed. The subjects underwent standard semen analysis, according to World Health Organization criteria. Semen samples obtained by masturbation after 3-5 days of sexual abstinence. Azoospermic men were evaluated according to the clinical parameters (testicular volume and structure, serum FSH levels and testicular biopsy) and only non-obstructive azoospermia patients were included in the study. The stress status of the childlessness in terms of sexual intercourse was scored by the Visual analogue scale (VAS) questionnaire. The scores before and after the birth of the child were compared. Patients with known systematic and psychiatric diseases, taking a medicine that may cause sexual dysfunction or the ones complaining of secondary infertility were excluded from the study. All the data provided were enrolled as part of a routinely clinical procedure and, ethical approval for the study was received from the Ethics Commitee. Written informed consent was obtained from patients who participated in this study.

\section{Statistical analysis}

All statistical analyses were performed with Statistical Package for the Social Science (SPSS Inc, Chicago, Illinois, USA) version 16.0. Normality of tests was analyzed with the Kolmogorov-Smirnov and Shapiro-Wilk tests.

The independent samples $t$ test was used for pairwise comparisons of parameters that were distributed normally, and the Mann Whitney U-test was used for parameters that were not distributed normally.

Differences were considered significant when $\mathrm{p}<0.05$.

\section{RESULTS}

The median age of the 193 male patients and their partners were 31 (range 23-48) and 27.2 (range 18-43) years, respectively. When the socio-cultural levels of male partners were examined, $59 \%$ of them were graduated from high school or university. Couples have been married for an average of 45 months (range 12-193) and the median period of infertility in these couples was 27 months (range 12-180). The average number of weekly frequency of coitus was around 2.5 (range 0.5-7). In this study, all of the cases consisted of primarily infertile couples, whereas a previously successful birth was not observed in any case. The mean number of treatments of couples prior to involvement in study was 0.6 (range 07) including oral medications, injectable fertility drugs, intrauterine insemination (IUI). The majority of men was in their first marriage (93\%), while a minority was in their second $(6 \%)$ or third marriage (1\%) (Table 1).

The results of the IIEF-15 questionnaire showed that 68 (35.2\%) men reported an overall ED. Of these, 31 (15.5\%) patients reported a mild ED (score 22-25), 25 (12.9\%) patients a mild to moderate (score 17-21), 9 (4.6\%) patients a moderate ED (score 11-16) and 3 (1.5 \%) patients have reported a severe form (score 6-10). Premature ejaculation was seen in $42(21.7 \%)$ patients according to PEDT score. In patients with a PEDT score $>8$ acquired and lifelong PE was reported by $56.2 \%$ and $43.8 \%$ of the patients, respectively. Erectile dysfunction was not seen in $50 \%$ of cases with PE, whereas PE was not observed in cases with severe ED.

Patients were divided into two groups according to presence of ED, (group 1: IIEF-15 ED score < 26 [n:68]; group 2: no $\mathrm{ED}$ [n:125]). The mean age of the patients was $33.1 \pm 6.55$ and $31.3 \pm 4.93$, respectively $(p=0.14)$. When comparing two groups there was no relationship between ED presence and education level but the education levels of men with severe and moderate ED were significantly lower than men with mild ED $(\mathrm{p}<0.05)$. Age of partners was $29.4 \pm 6.02$ and $27.5 \pm 5.15$, respectively, and weekly frequency of coitus was $2.1 \pm 0.84$ and $2.5 \pm 0.97$ respectively; differences were statistically significant ( $\mathrm{p}=0.053$ and 0.002 , respectively).

The mean number of treatment protocols applied to these couples was $2.1(0-4)$. One hundred and forty-one of the couples were treated with different treatment modalities such as oral drugs, injectable fertility drugs, intrauterine insemination (IUI). Successful pregnancies were obtained in 61 cases with assisted reproduction treatments after a mean of 10 months (3-18 months). Thirteen pregnancies were terminated due to different reasons. After the treatment period, the IIEF-15 test was repeated to 48 male partners of couples having children. We observed that IIEF-15 scores increased from 16 to 21 and that improvement in IIEF-15 scores was statistically

\section{Table 1.}

Sociodemographic and clinical characteristics of the subjects.

\begin{tabular}{|llc|}
\hline & & All patients (n: 193) \\
\hline Age (years) & & $31 \pm 4.2$ \\
\hline Partner's age (years) & Primary/secondary & $27.2 \pm 3.1$ \\
\hline Education (\%) & High/University & 51 \\
\hline Duration of marriage (months) & & $45(12-193)$ \\
\hline Number of marriage (\%) & First & 93 \\
& Second & 6 \\
& Third & 1 \\
\hline Duration of infertility (months) & & $27(12-180)$ \\
\hline Frequency of coitus (per week) & & $2.5(0.5-7)$ \\
\hline Erectile dysfunction $n(\%)$ & & $68(35.2)$ \\
\hline IIEF-15 score $n$ (\%) & No ED (26-30) & $125(64.8)$ \\
& Mild ED (22-25) & $31(15.5)$ \\
& Mild to Moderate (17-21) & $25(12.9)$ \\
& Moderate (11-16) & $9(4.6)$ \\
& Severe (6-10) & $3(1.5)$ \\
\hline Premature ejaculation $n$ (\%) & & $42(21.7)$ \\
\hline
\end{tabular}


Table 2

Comparison of stress levels of 48 male partners with childhood by using VAS questionnaire and ED and PE scores between childlessness and after having child.

\begin{tabular}{|lccc|}
\hline & Childlessness & Having child & $\mathbf{p}$ \\
\hline IIEF $(0-30)$ & 16 & 21 & 0.014 \\
\hline $\operatorname{PE}(0-20)$ & $6.3 \pm 2.1$ & $5.9 \pm 1.7$ & \\
\hline VAS $(0-10)$ & $5.2(3-10)$ & $4.1(0-8)$ & 0.02 \\
\hline
\end{tabular}

significant $(p=0.014)$. At the same time PE was questioned by PEDT in this group of patients, but no statistical difference was observed.

We evaluated the effect of having children on stress levels by VAS score. The Visual analogue scale (VAS) questionnaire was filled during the period of the use of assisted reproduction methods and in the next period after having children in order to evaluate the stress related to infertility. Their stress levels regarding sexual function in these two periods were scored as follows; 0: no stress, 10: very stressful. The mean VAS scores of male partners was $5.2(3-10)$ in treatment period while the same group mean score decreased to 4.1 (0-8) when they had children $(p=0.02)$. Statistical analysis showed a correlation between VAS and infertility like IIEF-15 (Table 2).

\section{Discussion}

Sexual dysfunctions are common problems in society. Premature ejaculation (29.3\%) is the main sexual displeasure in men in the general population; ED (14.5\%) follows as the second sexual health problem (17).

The prevalence of infertile couples ranges from $4 \%$ to $17 \%$ and sexual dysfunction may play an etiological role in these couples $(18,19)$. In one study, investigating presence of ED and PE in infertile men, Lotti et al. proved that both ED and PE were higher in them compared to fertile men (20). Another study in infertile men on in vitro fertilisation treatment, found no significant difference in infertile men in terms of ED, but these patients had more depressive mood (21). In a community research by Jain et al. premature ejaculation (66\%) was the most common problem and it was followed by erectile dysfunction (15\%), decreased libido (11\%) and orgasmic failure (8\%) among the infertile men (22). In a similar study by Lotti et al. erection and ejaculation status of infertile men were evaluated with IIEF and PEDT (12). They also researched psychological status with Middlesex Hospital Questionnaire (MHQ) and prostatitis symptoms with National Institutes of Health-chronic prostatitis symptom index (NIH-CPSI). Lotti et al. found lower rates of PE and ED (15.6\% and 17.8\%) than in our study $(21.7 \%$ and $34.7 \%$, respectively). Also depression was significantly associated with ED and they found a positive relationship between PE and prostatitis symptoms and phobic anxiety. According to these rates, it was obvious that men who had known that they were infertile, came across with more sexual problems such as PE and ED than fertile ones. These male partners, especially living in conservative societies, have a feeling of guiltiness and weakness so sexual fuction can not be fully performed by them (23).

There are many factors that can lead to the relation between infertility and sexual dysfunction such as age, race, religion, social status, employment status, level of education and previous paternity experience. In our study, we found that education levels of men with severe and moderate ED were significantly lower than in men with mild ED, similarly to the literature (23). Kizllay et al. found a close correlation between sperm parameters and sexual dysfunction in infertile couples. According to this study, poor sperm quality for count, morphology and motility were associated with severe ED in men and a parallel increase of female sexual dysfunction was observed (24). They also reported that worse sperm parameters accompanied declines in testosterone and IIEF scores. This comparison is not possible in terms of the azoospermia of all the cases in our study. However, known azoospermic group had the worst erectile function, higher PE prevalence, lower sexual desire, orgasmic function and general health condition among all infertile males (25).

In 2014, Bayar et al. examined sexual dysfunction before and after treatment in patients who received IVF treatment (26). IIEF form for male partners and FSFI form for female partners were used for determining sexual dysfunction. At the third month of IVF treatment, severity of sexual dysfunction increased to $72 \%$ of female partners and $48 \%$ of male partners. But in the subgroup analysis, there were not found significant differencies in erection status and PE. In our study we investigated the changes in ED and PE in case of couple's having a baby and we observed that IIEF-15 scores increased from 16 to 21 in male partners after having a baby after treatment. This improvement in IIEF-15 scores was statistically significant $(p=0.014)$. At the same time PE was questioned by PEDT in this group of patients, but no statistical difference was observed.

It had been observed that psychosexual problems rise at the maximum level when duration of childlessness lasted less than two years or more than eight years (14). Song et al. searched stress related to infertility and timed intercourse during fertile periods of male partners in infertile couples with VAS questionnaires (8). The mean VAS score of sexual relationship stress was significantly higher during fertile than non-fertile periods (3.4 vs 2.1). As the fecundity of healthy couples is about $20 \%$ per cycle, the wife and the environment create stress on men' sexual function during fertile periods (8). We investigated the effect of sexual relationship stress in infertile couples who had children after assisted reproduction procedures. To our knowledge, this is the first study to quantitatively investigate stress levels of male partners of infertile couples between the periods before after the birth of the child. In our study, after having child the VAS scores of male partners showed a statistically significant decrease to 4.1 from 5.2 during sexual intercourse. Furthermore this significant improvement was also observed on sexual functions. We observed a statistically significant increase in the mean IIEF-15 levels from 16 to 21 in case of having a baby although the same improvement was not seen in PE.

Our study has some limitations. Firstly, sexual functions of female partners were not investigated. A decrease in female sexual desire may cause a negative effect on sexual function of their male partners. Secondly, we did not eval- 
uate the psychological or somatic status of patients with psychiatric tests such as Middlesex Hospital Questionnaire (MHQ) or Short Form Health Survey 36 (SF-36) which can effect patients' sexual functions. Lastly, our study did not include fertile couples as a control group. Also the retrospective design of our study is an important bias.

Therefore, randomised prospective studies with large groups of infertile and fertile groups are needed to understand the main pathogenesis of male sexual dysfunction.

\section{Conclusions}

We aimed to investigate the prevalence of sexual dysfunction which included ED and PE in infertile men. Erectile dysfunction was more common in our patient group than the literature. We consider this as a repercussion of communicative obstacles encountered in conservative societies. Moreover another finding, contrasting the literature, was related to the assessment of $\mathrm{ED}, \mathrm{PE}$ and quantitative stress degree related to infertility (VAS scores) after having a baby through assisted reproduction treatments. We observed that having children is a reducing effect on sexual relationship stress. Sexuality can be deprived of its amusement and erotic value in case of a known infertility status. This condition may have negative affects on male sexual performance and it is closely related with occuring female sexual disorders. It should not be forgotten that infertile couples may have sexual dysfunction.

\section{REFERENCES}

1. Lee TY, Sun GH, Chao SC. The effect of an infertility diagnosis on the distress, marital and sexual satisfaction between husbands and wives in Taiwan. Hum Reprod 2001; 16:1762-7.

2. Nene UA, Jindal UN, Dhall GI. Infertility: A label of choice in the case of sexually dysfunctional couples. Patient Educ Couns 2005; 59:234-8

3. Brugh VM III, Lipshultz LI. Male factor infertility: Evaluation and management. Med Clin North Am 2004; 88:367-85.

4. Chevret M, Jaudinot E, Sullivan K, et al. Impact of erectile dysfunction (ED) on sexual life of female partners: Assessment with the Index of Sexual Life (ISL) Questionnaire. J Sex Marital Ther 2004; 30:157-72.

5. Speckens AE, Hengeveld MW, Lycklama a Nijeholt G, et al. Psychosexual functioning of partners of men with presumed nonorganic erectile dysfunction: Cause or consequence of the disorder? Arch Sex Behav 1995; 24:157-72.

6. Khademi A, Alleyassin A, Amini M, Ghaemi M. Evaluation of sexual dysfunction prevalence in infertile couples. J Sex Med. 2008; 5:1402-10

7. Lapane KL, Zierler S, Lasater TM, et al. Is a history of depressive symptoms associated with an increased risk of infertility in women? Psychosom Med 1995; 57:509-13.

8. Song SH, Kim DS, Yoon TK, et al. Sexual function and stress level of male partners of infertile couples during the fertile period. BJU Int. 2016; 117:173-6.

9. Hurwitz MB. Sexual dysfunction associated with infertility. A comparison of sexual function during the fertile and the nonfertile phase of the menstrual cycle. S Afr Med J. 1989; 76:58-61.

10. Shindel AW, Nelson CJ, Naughton CK, Mulhall JP. Premature ejaculation in infertile couples: Prevalence and correlates. J Sex Med. 2008; 5:485-91.
11. Cappelleri JC, Rosen RC, Smith MD, et al. Diagnostic evaluation of the erectile function domain of the International Index of Erectile Function. Urology. 1999; 54:346-51.

12. Lotti F, Corona G, Rastrelli G, et al. Clinical correlates of erectile dysfunction and premature ejaculation in men with couple infertility. J Sex Med. 2012; 9:2698-707.

13. Saleh RA, Ranga GM, Nelson DR, Agarwal A. Sexual dysfunction in men undergoing infertility evaluation: a cohort observation study. Fertil Steril. 2003; 79:909-12

14. Jindal UN, Dhall GI. Psychosexual problems of infertile women in India. Int J Fertil. 1990; 35:222-5.

15. Soykan A. The reliability and validity of Arizona sexual experiences scale in Turkish ESRD patients undergoing hemodialysis. Int J Impot Res. 2004; 16:531-4.

16. Symonds T, Perelman MA, Althof S, et al. Development and validation of a premature ejaculation diagnostic tool. Eur Urol. 2007; 52:565-73.

17. Yilmaz E, Zeytinci IE, Sari S, et al. Investigation of sexual problems in married people living in the center of Konya. Turk Psikiyatri Derg. 2010; 21:126-34.

18. Tuttelmann F, Nieschlag E. Classification of Andrological disorders. In: Nieschlag E, Behre HM, Nieschlag S, eds. Andrology. Male reproductive health and dysfunction. 3nd edition. Berlin: Springer, Verlag; 2010; 87-92.

19. Krausz C. Male infertility: Pathogenesis and clinical diagnosis. Best Pract Res Clin Endocrinol Metab. 2011; 25:271-85.

20. Lotti F, Corona G, Castellini G, et al. Semen quality impairment is associated with sexual dysfunction according to its severity. Human Reproduction. 2016; 31:2668-2680.

21. Ozkan B, Orhan E, Aktas N, Coskuner ER. Depression and sexual dysfunction in Turkish men diagnosed with infertility. Urology. 2015; 85:1389-1393.

22. Jain K, Radhakrishnan G, Agrawal P. Infertility and psychosexual disorders: relationship in infertile couples. Indian J Med Sci. 2000; $54: 1-7$.

23. Smith JF, Walsh TJ, Shindel AW, et al. Sexual, marital, and social impact of a man's perceived infertility diagnosis. J Sex Med. 2009; 6:2505-2515.

24. Kizllay F, Sahin M, Altay B. Do sperm parameters and infertility affect sexuality of couples? Andrologia. 2018; 50.

25. Lotti F, Corona $G$, Castellini $G$, et al. Semen quality impairment is associated with sexual dysfunction according to its severity. Hum Reprod. 2016; 31:2668-2680

26. Bayar U, Basaran M, Atasoy N, et al. Sexual dysfunction in infertile couples: evaluation and treatment of infertility. J Pak Med Assoc. 2014; 64:138-45.

\section{Correspondence}

Taha Numan Ylklmaz, MD (Corresponding Author) - numanyikilmaz@gmail.com Erdem Öztürk, MD - drerdemozturk@gmail.com

Halil Başar, MD - drhalilbasar@gmail.com ismail Selvi, MD - drismailselvi@gmail.com

Department of Urology, Ankara Dr. Abdurrahman Yurtaslan Oncology Training and Research Hospital, Ankara 06200, Turkey

Nurullah Hamidi, MD - drnurullahhamidi@gmail.com

Atatürk Training and Research Hospital, Ankara 06200, Department of Urology

Levent Peşkircioğlu, MD - drlevent@gmail.com

Baskent University Ankara Education and Research Hospital, Department of

Urology, Ankara, Turkey 